\title{
Enhancement of Oxygen Evolution Activity of Nickel Oxyhydroxide by Electrolyte Alkali Cations
}

\author{
Amanda C. Garcia, Thomas Touzalin, Celine Nieuwland, Nickson Perini, and \\ Marc T. M. Koper*
}

\begin{abstract}
Herein, the effect of the alkali cation $\left(\mathrm{Li}^{+}, \mathrm{Na}^{+}, \mathrm{K}^{+}\right.$, and $\left.\mathrm{Cs}^{+}\right)$in alkaline electrolytes with and without Fe impurities is investigated for enhancing the activity of nickel oxyhydroxide (NiOOH) for the oxygen evolution reaction (OER). Cyclic voltammograms show that Fe impurities have a significant catalytic effect on OER activity; however, both under purified and unpurified conditions, the trend in OER activity is $\mathrm{Cs}^{+}>\mathrm{Na}^{+}>\mathrm{K}^{+}>\mathrm{Li}^{+}$, suggesting an intrinsic cation effect of the OER activity on $\mathrm{Fe}$-free $\mathrm{Ni}$ oxyhydroxide. In situ surface enhanced Raman spectroscopy (SERS), shows this cation dependence is related to the formation of superoxo OER intermediate $\left(\mathrm{NiOO}^{-}\right)$. The electrochemically active surface area, evaluated by electrochemical impedance spectroscopy (EIS), is not influenced significantly by the cation. We postulate that the cations interact with the $\mathrm{Ni}-\mathrm{OO}^{-}$species leading to the formation of $\mathrm{NiOO}^{-}-\mathrm{M}^{+}$species that is stabilized better by bigger cations $\left(\mathrm{Cs}^{+}\right)$. This species would then act as the precursor to $\mathrm{O}_{2}$ evolution, explaining the higher activity.
\end{abstract}

$N$ ickel-based oxides have been extensively used as catalyst for the oxygen evolution reaction (OER $)^{[1-6]}$ because of their high intrinsic activity and stability in alkaline electrolyte. ${ }^{[7-12]}$ Understanding the processes and intermediates involved during OER on the nickel oxyhydroxide $(\mathrm{NiOOH})$ surface is of key importance for further improving their performance. The OER activity of $\mathrm{NiOOH}$ is known to depend on the electrolyte composition, in particular on the Fe content of the electrolyte, the nature of the cation in the electrolyte, and the $\mathrm{pH}$ of the electrolyte. ${ }^{[13-16]}$ The exposure of the $\mathrm{NiOOH}$ to $\mathrm{Fe}$ in the electrolyte changes the active-phase structure of $\mathrm{NiOOH}$. In spite of extensive in situ spectroscopic experi-

[*] Dr. A. C. Garcia, Dr. T. Touzalin, C. Nieuwland, Dr. N. Perini,

Prof. Dr. M. T. M. Koper

Leiden Institute of Chemistry

Leiden University

PO Box 9502, 2300 RA, Leiden (The Netherlands)

E-mail:m.koper@lic.leidenuniv.nl

Dr. N. Perini

Instituto de Química de São Carlos, Universidade de São Paulo Avenida Trabalhador São-Carlense 400, 13569-590 São Carlos, SP (Brazil)

Dr. A. C. Garcia

Current address: Department of Sustainable Process and Energy Systems, TNO, Leeghwaterstraat 44, 2628 CA Delft (The Netherlands)

(iD) Supporting information and the ORCID identification number(s) for the author(s) of this article can be found under:

https://doi.org/10.1002/anie.201905501. ments and detailed density functional theory (DFT) calculations, the exact nature of the active site in the $\mathrm{NiFe}$ oxyhydroxide is still under debate. ${ }^{[17-21]}$ While Bell et al. ${ }^{[19]}$ suggest that $\mathrm{Fe}$ are the active sites, Strasser et al. ascribe the high activity to a synergy at highly active Ni-Fe sites involving a negatively charged oxygenate ligand at Fe centers, activating adjacent Ni centers. ${ }^{[18,22]}$ However, also catalytically nonactive alkali and alkali earth cations in the electrolyte may impact on the activity of both $\mathrm{NiOOH}$ and $\mathrm{NiFeOOH}$. Michael et al. ${ }^{[15]}$ showed that in $\mathrm{CsOH}$ longer $\mathrm{Ni}-\mathrm{O}$ bonds in the $\mathrm{NiOOH}$ structure are observed. Toroker et al. have ascribed these effects to the intercalation of electrolyte cations into the layered structure of the nickel oxyhydroxide $^{[23]}$ which is known to undergo cation insertion upon the oxidation of $\mathrm{Ni}(\mathrm{OH})_{2}$ to $\mathrm{NiOOH} \cdot{ }^{[24-27]}$ Generally, these results show that the catalytic performance and active-phase structure of $\mathrm{NiOOH}$ catalysts can be tuned by changing the electrolyte cation and $\mathrm{Fe}$ contents in the electrolyte. ${ }^{[13-15,23]}$ Grimaud et al. ${ }^{[28]}$ also studied the interaction of cations with the NiOOH structure. They showed that tetraalkylammonium cations $\left(\mathrm{TTA}^{+}\right)$interact with the oxygen species formed upon deprotonation and these interactions only occur for $\mathrm{Fe}$ containing $\mathrm{NiOOH}$ catalysts.

Our group has shown that the activity of (iron-free) $\mathrm{NiOOH}$ for the OER is very sensitive to the $\mathrm{pH}$ of the electrolyte, with higher $\mathrm{pH}$ leading to higher activity. ${ }^{[16,29]}$ The $\mathrm{pH}$ dependence was explained by the degree of chemical deprotonation of $\mathrm{NiOOH}$, producing a negatively charged surface species [Equation (1)], which is responsible for the enhanced OER activity. Using in situ Raman spectroscopy, a peak in the $800-1150 \mathrm{~cm}^{-1}$ wavenumber range was attributed to "active oxygen" ${ }^{[30]}$ in the oxyhydroxide structure. We assigned this band to a superoxo surface species $\left(\mathrm{Ni}^{-} \mathrm{OO}^{-}\right)$ acting as the precursor for the $\mathrm{O}_{2}$ formation. We proposed a mechanism for the OER on $\mathrm{NiOOH}$ in which the deprotonation step (1) yields the active intermediate for $\mathrm{O}_{2}$ formation, consistent with the observed $\mathrm{pH}$ sensitivity.

$\mathrm{NiOOH} \rightleftharpoons \mathrm{Ni}-\mathrm{OO}^{-}+\mathrm{H}^{+}$

Since the acid-base equilibrium [Eq. (1)] yields a negatively charged species as the key intermediate, we expect it to be sensitive to the cation in the electrolyte. Using in situ Surface-Enhanced Raman spectroscopy (SERS) and electrochemical impedance spectroscopy (EIS), herein we present experimental evidence for the different stabilization of the active oxygen intermediate by different electrolyte cations. Our results lend further credit to our mechanism for OER on $\mathrm{NiOOH}$, and provide another example of how electrolyte 
cations influence the activity and selectivity of electrocatalytic reactions by their specific interaction with active surface intermediates. ${ }^{[31-34]}$

$\mathrm{NiOOH}$ electrocatalysts were prepared by electrochemically assisted precipitation of $\mathrm{Ni}(\mathrm{OH})_{2}$ on polycrystalline gold. ${ }^{[35]}$ For measurements on iron-free catalysts, the electrolytes were purified from $\mathrm{Fe}$ using the method described by Boettcher et al. ${ }^{[13]}$ See the Supporting Information for further experimental details.

We performed cyclic voltammetry $(\mathrm{CV})$ of $\mathrm{NiOOH}$ catalysts in both purified and unpurified alkali $\mathrm{MOH}$ electrolytes (with $\mathrm{M}^{+}=\mathrm{Cs}^{+}, \mathrm{K}^{+}, \mathrm{Na}^{+}$and $\mathrm{Li}^{+}$). First, a $\mathrm{CV}$ was recorded in Fe-free electrolyte until a stable profile was obtained. Next, the electrolyte was switched to the unpurified electrolyte containing traces of Fe. For each different electrolyte, a new $\mathrm{NiOOH}$ film was made in order to avoid any trace remnants of cations or Fe from the previous experiment in the structure of the nickel oxyhydroxide.

Figure 1 shows the current-potential curves for the four different cations in each electrolyte condition. For the unpurified electrolyte (solid lines in Figure 1), a clear enhancement effect of the cations is observed in terms of onset potential (ca. $100 \mathrm{mV}$ from $\mathrm{Li}^{+}$to $\mathrm{Cs}^{+}$) following the trend $\mathrm{Cs}^{+}>\mathrm{K}^{+}>\mathrm{Na}^{+}>\mathrm{Li}^{+}$(in agreement with the literature ${ }^{[15,23]}$ ), which in principle might suggest that the bigger cation leads to a higher OER activity. The CVs for the Fefree electrolyte (dotted lines in Figure 1) show that Fe impurities have a significant catalytic effect on OER activity, as is well known, ${ }^{[13,15]}$ so that the enhancement may be primarily related to different $\mathrm{Fe}$ contents of the different alkali electrolytes (see Table S1). However, even in the purified electrolyte, the trend in OER activity remains $\mathrm{Cs}^{+}>\mathrm{Na}^{+}>\mathrm{K}^{+}>$ $\mathrm{Li}^{+}$, suggesting that there is an intrinsic cation effect on the OER activity of $\mathrm{NiOOH}$.

We used in situ Raman spectroscopy to investigate the cation effect on the active oxygen intermediate. In agreement with previous work, SER spectra at potentials below $1.4 \mathrm{~V}$ versus the reversible hydrogen electrode (RHE) show two weak bands, one at about $450 \mathrm{~cm}^{-1}$ attributed to the $\alpha-\mathrm{Ni}(\mathrm{OH})_{2}$ and $\beta-\mathrm{Ni}(\mathrm{OH})_{2}$ phases and a second one at approximately $494 \mathrm{~cm}^{-1}$ which corresponds to the defective or distorted $\mathrm{Ni}(\mathrm{OH})_{2}$ phase. ${ }^{[37-39]}$ The $\mathrm{Ni}(\mathrm{OH})_{2} /$ $\mathrm{NiOOH}$ redox transition takes place at around $1.4 \mathrm{~V}$ vs. RHE, which gives rise to two well-defined SERS peaks at around $480 \mathrm{~cm}^{-1}$ and $560 \mathrm{~cm}^{-1}$, which can be assigned to the bending and stretching vibration modes, respectively, of $\mathrm{Ni}-\mathrm{O}$ in $\mathrm{NiOOH}$. As reported previously, these results indicate that the oxidation of $\mathrm{Ni}$ $(\mathrm{OH})_{2}$ occurs via a hydroxide-mediated process. ${ }^{[14-16,40,41]}$

Figure 2 shows spectra acquired at $1.7 \mathrm{~V}$ for all electrolytes. A cation dependence was observed for the bending and stretching vibrations both in unpurified (Figure $2 \mathrm{a}$ ) and purified (Figure $2 \mathrm{~b}$ ) electrolytes. The peaks related to $\mathrm{Ni}-\mathrm{O}$ bending in $\mathrm{CsOH}$ and $\mathrm{Fe}$-free $\mathrm{CsOH}$ exhibit lower Raman shifts (480.1 $\mathrm{cm}^{-1}$ and $481.2 \mathrm{~cm}^{-1}$, respectively) compared to the other cations. The highest Raman shifts for the $\mathrm{Ni}-\mathrm{O}$ bending mode were found for to $\mathrm{LiOH}$ and $\mathrm{Fe}$-free $\mathrm{LiOH}$ at

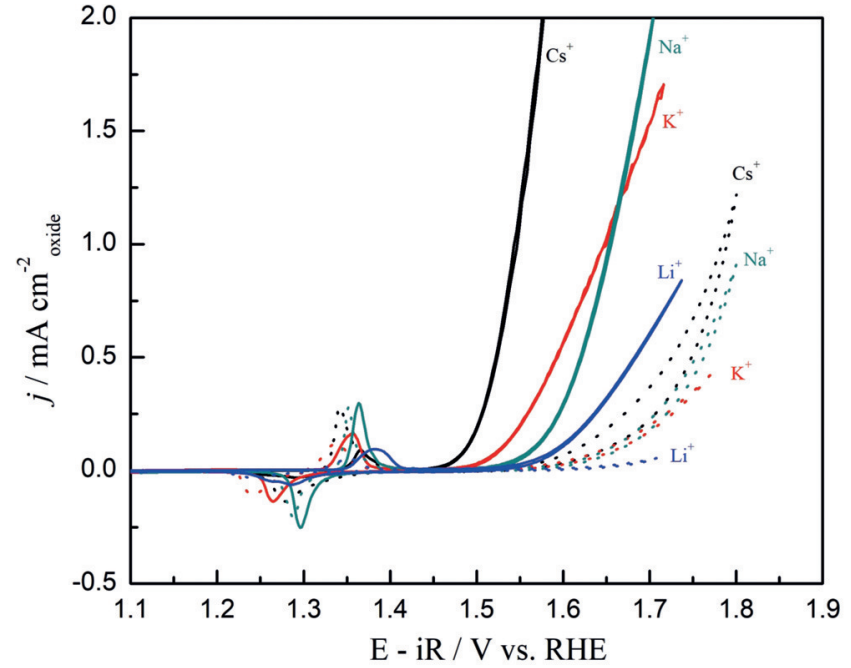

Figure 1. Cyclic voltammograms of $\mathrm{NiOOH}$ at $\mathrm{pH} 13$ in the region of oxygen evolution in different electrolytes. Solid lines: unpurified electrolytes; dotted lines: electrolytes purified by the method specified in the text. Measurements were performed under rotation of the working electrode (1600 rpm). Cyclic voltammograms were collected at $0.010 \mathrm{Vs}^{-1}$. Currents normalized by specific capacitance (see Supporting Information). ${ }^{[36]}$

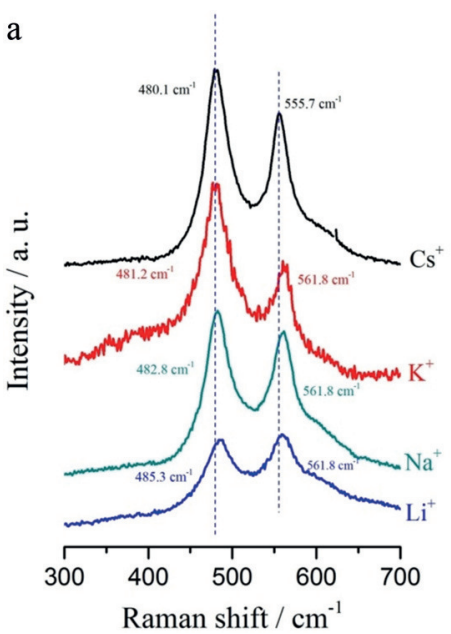

b

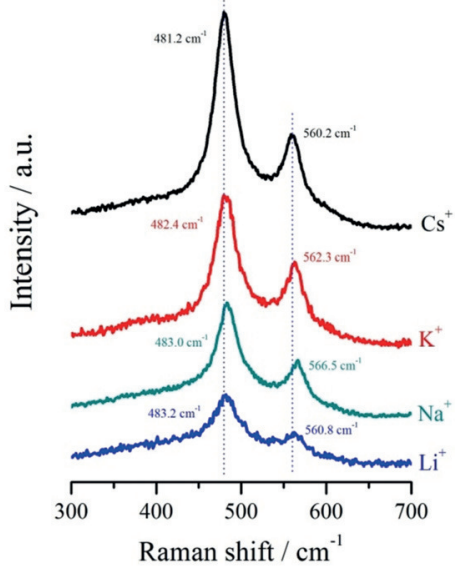

Figure 2. In situ Raman spectra of $\mathrm{NiOOH}$ obtained at $1.7 \mathrm{~V}$ in $\mathrm{pH} 13$ in the presence of different electrolyte cations. a) Unpurified electrolyte and b) purified electrolyte.

$485.3 \mathrm{~cm}^{-1}$ and $483.2 \mathrm{~cm}^{-1}$, respectively. Similar cation-dependent shifts are observed for the $\mathrm{Ni}-\mathrm{O}$ stretching mode near $580 \mathrm{~cm}^{-1}$. These results are in good agreement with earlier results by Michael et al. who have related this longer $\mathrm{Ni}-\mathrm{O}$ bond to the higher OER activity observed. ${ }^{[15]}$ A lower Raman shift can indeed be induced by a longer $\mathrm{M}-\mathrm{O}$ bond. ${ }^{[42]}$

Of specific interest in this communication is the cation effect on the SERS feature at $800-1150 \mathrm{~cm}^{-1}$, the "active oxygen" which we have previously identified with the active intermediate for the oxygen evolution reaction (experiments in ${ }^{18} \mathrm{O}$-labelled water indeed confirmed the oxygen-related nature of this band). ${ }^{[16]}$ Figure 3 shows the SER spectra in the 

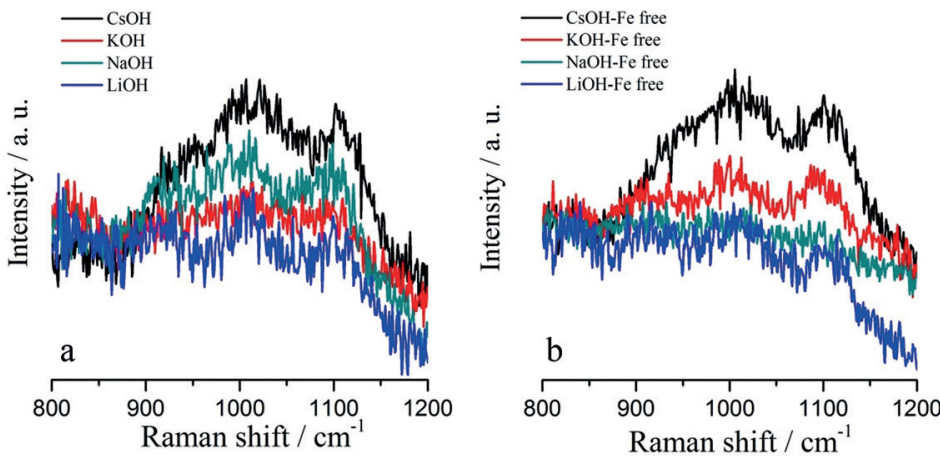

model recently put forward by Grimaud et al., ${ }^{[28]}$ who studied the influence of large organic tetra-alkyl ammonium cations, on the OER activity of $\mathrm{NiOOH}$ and $\mathrm{Ni}(\mathrm{Fe}) \mathrm{OOH}$ catalysts. They suggested that the strong interaction with organic cations actually blocks the active site and lowers the OER activity, specifically of the $\mathrm{Ni}(\mathrm{Fe}) \mathrm{OOH}$ catalyst.

Since alkali cations are known to intercalate in the $\mathrm{NiOOH}$ layers, an alternative interpretation of the cation effect would be to view Equation (2) as an effective exfoliation reaction, as suggested by Toroker et al. ${ }^{[44-46]}$ Since exfoliation should lead to a more open structure with a higher surface area, we measured the electrochemically active surface area in the different electrolytes.

Watzele and Bandarenka recently suggested the "active oxygen" wavenumber region (800-1150 $\left.\mathrm{cm}^{-1}\right)$ for the different unpurified and purified electrolytes.

The spectra exhibit two main broad peaks at 1010 and $1110 \mathrm{~cm}^{-1}$ for all cations with similar relative intensities independent of the Fe content of the electrolyte (purified and/ or unpurified). The Raman peak at $1100 \mathrm{~cm}^{-1}$ in $\mathrm{CsOH}$ and Fe-free $\mathrm{CsOH}$ is however broader and exhibits in both cases a shoulder in the $930-950 \mathrm{~cm}^{-1}$ region with a higher intensity compared to smaller alkali cations. Even if this vibration mode cannot be clearly assigned, it underscores a different interaction of $\mathrm{Cs}^{+}$with $\mathrm{NiOOH}$ compared to the other cations. Both this observation and the red shift observed with smaller cations in the $\mathrm{Ni}-\mathrm{O}$ bending and stretching mode, highlight the importance of the interaction of alkali cations with the $\mathrm{Ni}-\mathrm{OO}^{-}$species that might lead to a different stabilization of the superoxo intermediate.

To probe the effect of $\mathrm{Fe}$ in the electrolyte, we compared the spectra in the "active oxygen" wavenumber region for $\mathrm{CsOH}$ with different $\mathrm{Fe}$ contents (Supporting Information-1b). The spectra showed that the intensity of the superoxo peak is only slightly influenced by increasing $\mathrm{Fe}$ content. This result suggests that the active $\mathrm{NiOOH}$ intermediate in the absence of $\mathrm{Fe}$, is not the key active site in the $\mathrm{NiFeOOH}$ catalyst, in agreement with the conclusions drawn by Bell et al. ${ }^{[19]}$ and Strasser et al. ${ }^{[18,22]}$
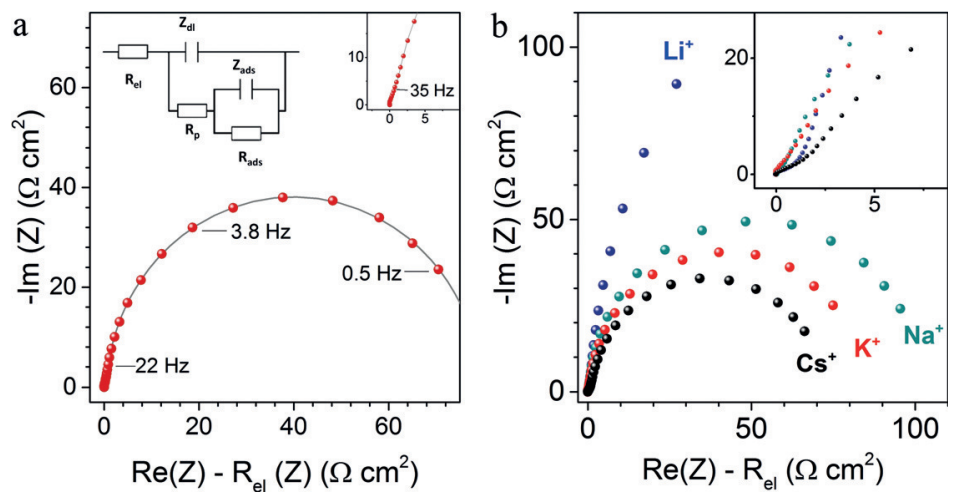

Figure 4. Typical Nyquist representations of electrochemical impedance spectra of $\mathrm{NiOOH}$ at $1.6 \mathrm{~V}$ vs. RHE and $\mathrm{pH}$ 13. a) El spectrum of a $\mathrm{NiOOH}$ layer in $\mathrm{KOH}$ : experimental spectrum (red dots) and fitted spectrum (gray line) using the Armstrong-Henderson equivalent circuit shown. The inset displays the high frequency region; b) El spectra plotted for different alkali cations (inset: highfrequency region).

Therefore, our results cannot identify the active intermediate in $\mathrm{NiFe}$ oxyhydroxide.

We interpret the cation dependence of the OER activity by assuming that the cation interacts with the $\mathrm{Ni}-\mathrm{OO}^{-}$species in Equation (1), leading to a modified acid-base reaction as expressed in Equation (2):

$\mathrm{NiOOH}+\mathrm{M}^{+} \rightleftharpoons \mathrm{NiOO}^{-}-\mathrm{M}^{+}+\mathrm{H}^{+}$

The results displayed in Figure 3 would imply that bigger cations (in this case $\mathrm{Cs}^{+}$) provide a better stabilization to the $\mathrm{Ni}-\mathrm{OO}^{-}-\mathrm{M}^{+}$intermediate. This species acts as the precursor to $\mathrm{O}_{2}$ evolution, explaining the higher activity. The exact nature of this cation stabilization should be the subject of further computational study (see, e.g., Refs. [34,43]). The model expressed in Equation (2) is subtly different form the representative EI spectrum is given in Figure 4 a with the fitted spectrum exhibiting two frequency regimes: the highfrequency regime is characterized by a time constant $\tau_{1}=$ $C_{\mathrm{dl}} \cdot R_{\mathrm{p}}$ (electrochemical double-layer) and the low-frequency time constant $\tau_{2}=R_{\text {ads }} \cdot C_{\text {ads }}$ is characteristic of the adsorption process (Faradaic process). Figure $4 \mathrm{~b}$ displays four EI spectra recorded at $1.6 \mathrm{~V}$ for the four different electrolytes: the same two-frequency behavior is observed in all electrolytes and as expected the low frequency impedance increases with the same trend observed for OER activity in $\mathrm{CVs} \mathrm{Li}>\mathrm{Na}>\mathrm{K}>$ Cs. In the Supporting Information, we show that the adsorption capacitance increases linearly with the amount of nickel deposited $\left(C_{\mathrm{ads}}=6.6 \mu \mathrm{Fnmol}^{-1}{ }_{\mathrm{Ni}}\right.$ in LiOH, $\left.\mathrm{pH} 13\right)$ on the gold electrode, indicating that active sites are located within the bulk material. This trend is also accompanied by an 
increase of the OER activity within the investigated range of $\mathrm{NiOOH}$ thickness (Figure S2). Therefore, the adsorption capacitance values are normalized by the amount of nickel atoms deposited on the electrode. Table 1 gives the adsorption capacitance values in the four different electrolytes.

Table 1: Adsorption and double-layer capacitances measured in different electrolytes at $1.6 \mathrm{~V}$ versus $\mathrm{RHE}, \mathrm{pH} 13$.

\begin{tabular}{lll}
\hline Cation & $C_{\text {ads }}\left(\mu \mathrm{F} \mathrm{nmol}{ }^{-1}\right)$ & $C_{\mathrm{dl}}\left(\mu \mathrm{F} \mathrm{cm}{ }^{-2}\right)$ \\
\hline $\mathrm{Li}^{+}$ & $7.2 \pm 1.1$ & $216 \pm 66$ \\
$\mathrm{Na}^{+}$ & $6.0 \pm 1.9$ & $291 \pm 36$ \\
$\mathrm{~K}^{+}$ & $4.5 \pm 2.0$ & $278 \pm 15$ \\
$\mathrm{Cs}^{+}$ & $6.7 \pm 2.5$ & $289 \pm 12$ \\
\hline
\end{tabular}

Although the values are rather dispersed around the average value of approximately $6.1 \mu \mathrm{Fnmol}^{-1}{ }_{\mathrm{Ni}}$ within the error bars they are found not to be significantly influenced by the nature of the cation, suggesting that the alkali cations do not measurably increase the electroactive area of the electrode. Moreover, the double-layer capacitance at $1.6 \mathrm{~V}$ is in the 200 $300 \mu \mathrm{F} \mathrm{cm}^{-2}$ range, which is consistent with values reported previously for similar nickel oxyhydroxide electrodes, ${ }^{[48,50]}$ but they are not significantly affected by the alkali cations in the electrolyte. If there is any trend in Table 1, the trend has no correlation with the activity trend observed in Figure 1.

We conclude that the insertion of alkali cations and their interaction with the $\mathrm{NiOOH}$ sheets does not yield a measurable change in the electroactive surface area. We therefore postulate that the interaction of the cation with the charged intermediate and its resulting stabilization plays a major role in the promoting effect of large alkali cations on the OER activity. Future computational investigations should take into account the changes in the structure induced by alkali cations insertion $^{[27]}$ and their interaction with the (deprotonated) $\mathrm{NiOOH}$ active sites.

In conclusion, we have shown that there is an intrinsic cation effect on the OER activity of Fe-free Ni oxyhydroxide catalysts. This cation effect is related to the interaction of the cation with the active oxygen species within the threedimensional oxide structure detected by using SERS, and not by an increase in surface area caused by cation-induced exfoliation. This explanation agrees with a model for the $\mathrm{pH}$ dependence of the NiOOH OER activity, which assumes the existence of a negatively charged reaction intermediate, the stability of which is sensitive to interaction with cations in the electrolyte. Our work thereby provides a rationalization for activating $\mathrm{Ni}(\mathrm{Fe}) \mathrm{OOH}$ catalysts for high OER activity by electrolyte cations. Further work will be needed to elucidate the exact nature of this stabilization.

\section{Acknowledgements}

A.C.G., T.T., and M T.M.K. thank the European Union for supporting this work under the a-leaf project (732840-ALEAF). C.N. thanks the Holland Research School of Molecular Chemistry (HRSMC) and N.P. acknowledges the
São Paulo Research Foundation (FAPESP-project number 17/05040-1) for a scholarship.

\section{Conflict of interest}

The authors declare no conflict of interest.

Keywords: cations - electrocatalysis - nickel oxyhydroxide · oxygen evolution reaction (OER) - Raman spectroscopy

How to cite: Angew. Chem. Int. Ed. 2019, 58, 12999-13003 Angew. Chem. 2019, 131, 13133-13137

[1] D. A. Corrigan, J. Electrochem. Soc. 1987, 134, 377.

[2] F. Song, X. Hu, Nat. Commun. 2014, 5, 1 -9.

[3] P. Oliva, J. F. Laurent, B. A. Daney, C. Delmas, J. J. Braconnier, F. Fievet, D. Bordeaux, M. Figlarz, P. Jussieu, A. De Guibert, et al., J. Power Sources 1982, 8, 229-255.

[4] O. Diaz-Morales, I. Ledezma-Yanez, M. T. M. Koper, F. CalleVallejo, ACS Catal. 2015, 5, 5380-5387.

[5] C. C. L. McCrory, S. H. Jung, J. C. Peters, T. F. Jaramillo, J. Am. Chem. Soc. 2013, 135, $16977-16987$.

[6] B. Kim, A. Oh, M. K. Kabiraz, Y. Hong, J. Joo, H. Baik, S.-I. Choi, K. Lee, ACS Appl. Mater. Interfaces 2018, 10, 1011510122.

[7] C. Dette, M. R. Hurst, J. Deng, M. R. Nellist, S. W. Boettcher, ACS Appl. Mater. Interfaces 2019, 11, 5590-5594.

[8] D. A. Corrigan, S. L. Knight, J. Electrochem. Soc. 1989, 136, 613.

[9] J. Desilvestro, D. A. Corrigan, M. J. Weaver, J. Electrochem. Soc. 1988, 135,885 .

[10] B. S. Yeo, A. T. Bell, J. Phys. Chem. C 2012, 116, 8394-8400.

[11] D. K. Bediako, B. Lassalle-Kaiser, Y. Surendranath, J. Yano, V. K. Yachandra, D. G. Nocera, J. Am. Chem. Soc. 2012, 134, $6801-6809$.

[12] K. Zeng, D. Zhang, Prog. Energy Combust. Sci. 2010, 36, $307-$ 326.

[13] L. Trotochaud, S. L. Young, J. K. Ranney, S. W. Boettcher, J. Am. Chem. Soc. 2014, 136, 6744-6753.

[14] M. W. Louie, A. T. Bell, J. Am. Chem. Soc. 2013, 135, 1232912337.

[15] J. D. Michael, E. L. Demeter, S. M. Illes, Q. Fan, J. R. Boes, J. R. Kitchin, J. Phys. Chem. C 2015, 119, 11475-11481.

[16] O. Diaz-Morales, D. Ferrus-Suspedra, M. T. M. Koper, Chem. Sci. 2016, 7, 2639-2645.

[17] Y. Gorlin, T. Jaramillo, J. Am. Chem. Soc. 2010, 132, 1361213614.

[18] M. Görlin, J. F. De Araújo, H. Schmies, D. Bernsmeier, S. Dresp, M. Gliech, Z. Jusys, P. Chernev, R. Kraehnert, H. Dau, P. Strasser, J. Am. Chem. Soc. 2017, 139, 2070-2082.

[19] D. Friebel, M. W. Louie, M. Bajdich, K. E. Sanwald, Y. Cai, A. M. Wise, M. J. Cheng, D. Sokaras, T. C. Weng, R. AlonsoMori, et al., J. Am. Chem. Soc. 2015, 137, 1305-1313.

[20] M. Gao, W. Sheng, Z. Zhuang, Q. Fang, S. Gu, J. Jiang, Y. Yan, J. Am. Chem. Soc. 2014, 136, $7077-7084$.

[21] V. Tripkovic, H. A. Hansen, T. Vegge, ACS Catal. 2017, 7, 8558 8571.

[22] M. Görlin, P. Chernev, J. F. De Araújo, T. Reier, S. Dresp, B. Paul, R. Krähnert, H. Dau, P. Strasser, J. Am. Chem. Soc. 2016, $138,5603-5614$.

[23] J. Zaffran, M. B. Stevens, C. D. M. Trang, M. Nagli, M. Shehadeh, S. W. Boettcher, M. C. Toroker, Chem. Mater. 2017, 29, $4761-4767$.

[24] P. Bernard, C. Gabrielli, M. Keddam, H. Takenouti, J. Leonardi, P. Blanchard, Electrochim. Acta 1991, 36, 743-746. 
[25] G. T. Cheek, W. E. O'Grady, J. Electroanal. Chem. 1997, 421, 173-177.

[26] I. C. Faria, R. Torresi, A. Gorenstein, Electrochim. Acta 1993, 38, $2765-2771$.

[27] M. Wehrens-Dijksma, P. H. L. Notten, Electrochim. Acta 2006, 51, 3609-3621.

[28] C. Yang, O. Fontaine, J. Tarascon, A. Grimaud, Angew. Chem. Int. Ed. 2017, 56, 8652-8656; Angew. Chem. 2017, 129, 87788782.

[29] B. J. Trześniewski, O. Diaz-Morales, D. A. Vermaas, A. Longo, W. Bras, M. T. M. Koper, W. A. Smith, J. Am. Chem. Soc. 2015, 137, 15112-15121.

[30] M. Merrill, M. Worsley, A. Wittstock, J. Biener, M. Stadermann, J. Electroanal. Chem. 2014, 717-718, 177-188.

[31] L. D. Chen, M. Urushihara, K. Chan, J. K. Nørskov, ACS Catal. 2016, 6, 7133-7139.

[32] I. T. McCrum, M. J. Janik, J. Phys. Chem. C 2016, 120, 457-471.

[33] E. Pérez-Gallent, G. Marcandalli, M. C. Figueiredo, F. CalleVallejo, M. T. M. Koper, J. Am. Chem. Soc. 2017, 139, 1641216419.

[34] X. Chen, I. T. McCrum, K. A. Schwarz, M. J. Janik, M. T. M. Koper, Angew. Chem. Int. Ed. 2017, 56, 15025-15029; Angew. Chem. 2017, 129, 15221-15225.

[35] J. House, Inorganic Chemistry, 2nd ed., Academic Press, San Diego, 2013.

[36] S. Trasatti, O. A. Petrii, J. Electroanal. Chem. 1992, 327, 353 376.

[37] Y. L. Lo, B. J. Hwang, Langmuir 1998, 14, 944-950.

[38] P. Hermet, L. Gourrier, J.-L. Bantignies, D. Ravot, T. Michel, S. Deabate, P. Boulet, F. Henn, Phys. Rev. B 2011, 84, 235211.
[39] H. B. Li, M. H. Yu, F. X. Wang, P. Liu, Y. Liang, J. Xiao, C. X. Wang, Y. X. Tong, G. W. Yang, Nat. Commun. 2013, 4, 1894.

[40] M. E. G. Lyons, A. Cakara, P. O'Brien, I. Godwin, R. L. Doyle, Int. J. Electrochem. Sci. 2012, 7, 11768-11795.

[41] M. E. G. Lyons, R. L. Doyle, M. P. Brandon, Phys. Chem. Chem. Phys. 2011, 13, 21530-21551.

[42] F. D. Hardcastle, I. E. Wachs, J. Raman Spectrosc. 1990, 21, 683 691.

[43] I. McCrum, X. Chen, K. A. Schwarz, M. J. Janik, M. T. M. Koper, J. Phys. Chem. C 2018, 122, 16756-16764.

[44] J. Zaffran, M. Nagli, M. Shehadeh, M. C. Toroker, Theor. Chem. Acc. 2018, 137, 3.

[45] J. Zaffran, M. C. Toroker, ChemElectroChem 2017, 4, 2764 2770.

[46] M. Nagli, M. C. Toroker, J. Chem. Phys. 2018, 149, 141103.

[47] S. Watzele, A. S. Bandarenka, Electroanalysis 2016, 28, 2394 2399.

[48] M. E. G. Lyons, M. P. Brandon, Int. J. Electrochem. Sci. 2008, 3, $1386-1424$.

[49] R. D. Armstrong, M. Henderson, J. Electroanal. Chem. Interfacial Electrochem. 1972, 39, 81-90.

[50] P. Chakthranont, J. Kibsgaard, A. Gallo, J. Park, M. Mitani, D. Sokaras, T. Kroll, R. Sinclair, M. B. Mogensen, T. F. Jaramillo, ACS Catal. 2017, 7, 5399-5409.

Manuscript received: May 6, 2019

Accepted manuscript online: June 28, 2019

Version of record online: July 25, 2019 